\title{
Network constraints in techno-economic energy system models: towards more accurate modeling of power flows in long-term energy system models
}

\author{
Christoph Nolden • Martin Schönfelder • \\ Anke Eßer-Frey • Valentin Bertsch • Wolf Fichtner
}

\begin{abstract}
Power systems are subject to extensive structural changes as a result of the fact that the share of renewable energies in power supply will increase significantly within the next decades. This requires the transport of large amounts of electricity, e.g. from the North Sea to the large load centres. Moreover, the decentralized installations for the generation of electricity (e.g. PV) need to be integrated in the lower voltage power grids without violating net-safety constraints. As a consequence, the grid load in the system will rise to an extent that is hardly manageable with existing power grid capacities. Therefore, while mostly neglected to date, the importance of considering the power grid in energy system models increases significantly. Within this paper, different examples will be given how network constraints can be considered in techno-economic energy system models with a focus on capacity expansion planning and a long-term time horizon. Firstly, a multi-period linear optimization model will be presented, which comprises the system equations for power generation and transmission. The latter is analyzed with the help of a DC power flow model. Secondly, the usage of an AC power flow modeling tool for a detailed representation of the medium and low voltage power grid will be described. Finally, we will present an illustrative example application of a new mathematical approach for grid modeling in techno-economic energy system models.
\end{abstract}

Keywords Energy systems analysis - Technical network constraints · Expansion planning $\cdot$ Integration of renewables $\cdot$ Load flow models $\cdot$ Nodal pricing

C. Nolden $(\varangle) \cdot$ M. Schönfelder · A. Eßer-Frey · V. Bertsch · W. Fichtner Karlsruhe Institute of Technology (KIT), Institute for Industrial Production (IIP), Chair of Energy Economics, Hertzstraße 16, 76187 Karlsruhe, Germany e-mail:nolden@kit.edu 


\section{Introduction}

The rearrangement of the energy system as a result of the ambitious targets concerning the expansion of renewable energy brings about new challenges, for both the electricity grid and the electricity supply. With the integration of renewable energies in the electricity generating system the function of the high voltage transmission grid (220/380 $\mathrm{kV}$ ) has changed. Before the liberalization of the energy markets and the EEG coming into effect, power plants were constructed near load centers. There was no need for electricity to be transported over long distances. A former function of the transmission grid was to serve as a backup system in case of plant failures [1].

Since then, a growing number of distributed renewable plants have been installed. In general, they are not necessarily located near the load centers but in places where large amounts of renewable energy are easily accessible. Nowadays, this leads to an increasing regional imbalance between production and consumption of electric energy, resulting in the need to transport large amounts of energy over very long distances. The local production of electricity from renewable sources may soon reach a level where the imbalances can no longer be covered by the transmission grid, especially in times of very high renewable production.

But not only the transmission grids are facing overloads. Medium and low voltage grids are also facing such challenges as a result of the strongly increasing share of fluctuating renewable energy generation. This is true for large wind parks in the $110 \mathrm{kV}$ level as well as for photovoltaic plants, ranging from a few $\mathrm{kW}$ to several MW. Besides the overloading of individual grid sections, another challenge can be the reversion of the load flow. All grids were originally constructed under the assumption of a central feeding and local loads, meaning, that the energy was only flowing from the higher to the lower voltage levels, e.g. from a transformer station to households. With the increased integration of renewable energies in the lower voltage levels, this has changed significantly. Backfeedings from a lower to a higher voltage level can be observed more often. Just as well this phenomenon can occur only in the low voltage grid, changing the flow direction on single lines and lowering the total load at the transformer station. In general, it is therefore important to adjust the grid protection equipment to bidirectional power flows. Overall, this necessitates increasing investments in grid infrastructure [2].

Besides grid extensions or load management approaches on the demand or supply side, a possible solution to these problems could be energy storages. In times of high energy production from renewable sources (e.g. strong winds or high solar radiation at noon), they could store exceeding electricity and re-feed it in times of low local production, leading to an overall decreased volatility of the residual load. In future, one promising approach might be the integration of electric vehicles in the grid, which, in large numbers, could soothe the problem. However, as even a large number of electric vehicles would not be a final solution, other storage technologies are needed. Unfortunately none of the currently discussed storage technologies is yet available in industrial standards or at reasonable investments. In short and middle term it will therefore be necessary to resort to alternative measures to avoid grid bottlenecks.

Altogether, every level of the electricity grid is facing essential changes. Given the fact that these changes vary in the different voltage levels, it is clear that a differential analysis and modeling of transportation and distribution grids is necessary. The 
combination of the different modeling types in one integrated model would be very desirable, but is limited due to several reasons. Firstly, the number of variables related to an integrated grid model would negatively affect the solvability and computing time. Secondly, the required data is usually poorly maintained or not existent. Finally, distribution grids differ on a large scale in terms of structure, installed equipment, or number of installed renewable plants, i.e. a separate examination of the different grids providing a deeper insight to specific questions can be advisable.

Therefore, after a basic overview on load flow modeling is given in Sect. 2 with special respect to the problems to be solved for the integration in energy system models, three different approaches are presented in this paper in order to address the different challenges described above. Firstly, the linear optimization model PERSEUS-NET is presented in Sect. 3. The model comprises a locational marginal pricing approach based on a DC load flow model to optimize regional power plant investment and unit commitment considering the transmission grid. Secondly, the application of the commercially available grid calculation tool NEPLAN is described in Sect. 4. The tool is used to analyze an urban distribution grid in Baden-Wuerttemberg (South Germany). In order to examine individual challenges in the distribution grid, the tool is more suited than PERSEUS-NET since it provides AC load flow analysis capabilities, which is highly important in distribution grids. However, the tool does not allow for economic analyses. Therefore, a first application of a new approach of direct integration of an AC load flow model into an energy system model is presented in Sect. 5. The mathematically challenging integration of an AC load flow model is described and the suitability of the concept is discussed (the complete mathematical definition is given in our previous publication [3]). Finally, conclusions are drawn regarding the applicability of the different approaches in the various circumstances. The paper ends with a short outlook on future research possibilities.

\section{Energy system modeling with respect to technical network constraints}

Energy system models originally serve to analyze the complex interplay of numerous players which try to satisfy the energy demand with different technologies. Especially the ability of taking all occuring interdependencies into account leveraged the success of energy system models in research and policy advice.

Regarding electric energy, one main assumption of standard models is, that the transport from supply to demand does not need to be restricted because the power grid has a sufficient capacity. This assumption led to the description as copperplate regions. Furthermore, electricity production is assumed to be controllable which, in reality, facilitates the handling of possible network congestions. However, ongoing structural changes as described in Sect. 1 necessitate the endogenous consideration of technical network constraints [4].

In the following, several different methods of load flow modeling are described. The most accurate approach is the so called alternating current (AC) load flow modeling, which comprises both active and reactive power as well as all network losses. Due to the mathematically challenging characteristics of AC models, literature reveals several simplified methods which are used both in electrical engineering and recent energy 
Table 1 Overview of selected OPF approaches in energy system analysis (based on [3])

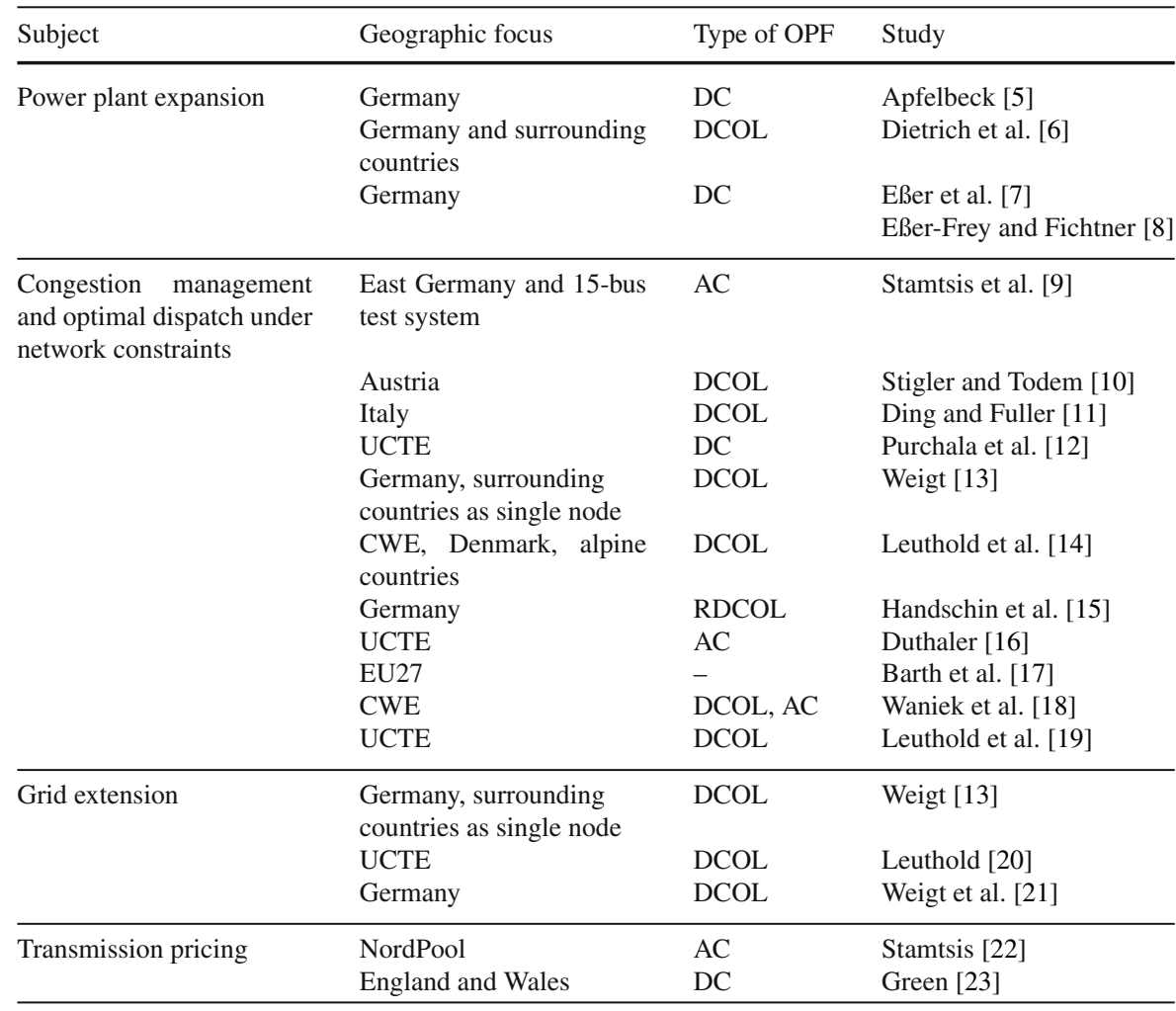

system models. The most frequently used is the so called direct current (DC) approach that linearizes power flow equations and therefore is easy to be integrated into linear energy system models. Table 1 gives an overview of realized models which integrate optimal power flow (OPF) methods in energy system analysis.

\subsection{Alternating current load flow models}

The technically accurate calculation of power flows in electrical networks necessitates the calculation of both the active and reactive power. The apparent power $S_{k}$ at node $k$ is a complex number. It comprises the real part $P_{k}$ (the active power) and the complex part $Q_{k}$ (the reactive power). The apparent power at node $k$ is calculated as the product of voltage $U_{k}$ and current $I_{k}$ at node $k$ as shown in Eq. (1). The following formal description is mainly based on [15] and [3].

$$
\underline{S_{k}}={\underline{U_{k}}}^{*}=P_{k}+j Q_{k}
$$

The real and reactive power at node $k$ equal the sums of active and reactive power flows on lines adjacent to node $k$ (see Eqs. (2) and (3)). 


$$
\begin{aligned}
P_{k} & =\sum_{m \in N_{k}} p_{k m} \\
Q_{k} & =\sum_{m \in N_{k}} q_{k m}
\end{aligned}
$$

The essential factor in power grid modeling is the apparent power flow over a line $s_{k m}$ from node $k$ to $m$. It is a function of the voltage levels, the phase angle difference of the node voltages, and the line and network characteristics. The physical laws have to be respected because the transport of electric energy can not be modeled like the transport of normal commodities. Equations (5) and (6) describe the underlying nonlinear relationships which are merged in (4) for the calculation of $s_{k m}$.

$$
\begin{aligned}
\underline{s_{k m}=} & g_{k m} U_{k}^{2}-g_{k m} U_{k} U_{m} \cos \theta_{k m} \\
& -b_{k m} U_{k} U_{m} \sin \theta_{k m}+j\left(b_{k m} U_{k} U_{m} \cos \theta_{k m}\right. \\
& \left.-g_{k m} U_{k} U_{m} \sin \theta_{k m}-U_{k}^{2}\left(b_{k m}^{q}+b_{k m}\right)\right) \\
p_{k m}= & g_{k m} U_{k}^{2}-g_{k m} U_{k} U_{m} \cos \theta_{k m} \\
& -b_{k m} U_{k} U_{m} \sin \theta_{k m} \\
q_{k m}= & b_{k m} U_{k} U_{m} \cos \theta_{k m}-g_{k m} U_{k} U_{m} \sin \theta_{k m} \\
& -U_{k}^{2}\left(b_{k m}^{q}+b_{k m}\right)
\end{aligned}
$$

The line characteristics describe the transfer capability of the line. They comprise the conductance $g_{k m}$, the inductive susceptance $b_{k m}$, and the capacitive susceptance $b_{k m}^{q}$ of the line.

The power flows at all other nodes and power lines of the network can be calculated analogously. Furthermore, the active and reactive power flows over a line $\mathrm{km}$ have to respect the thermal line limitations $C_{k m}$ as shown in Eq. 7.

$$
\underline{s_{k m}}=\sqrt{p_{k m}^{2}+q_{k m}^{2}} \leq C_{k m}
$$

The resulting power flow equations, which have to be integrated into the energy system model, are non-linear and neither convex nor concave. Thus, an iterative, computationally expensive approach has to be used to solve the problem which is today, in general, the Newton-Raphson Method (cf. e.g. [24]). Its convergence performance depends largely on the starting values chosen [24]. Especially for large systems, the duration of the solution process can be very long.

\subsection{Direct current load flow models}

A common simplification of alternating current load flow models are the so called direct current (DC) load flow models. Several assumptions reduce the equations aiming at the goal of linearity. The approximations that are generally made in DC models to simplify the solution process are (cf. e.g. $[15,24,25])$ : 
1. The voltage angle differences $\theta_{k m}$ are assumed to be very small, so that $\cos \theta_{k m} \approx 1$ and $\sin \theta_{k m} \approx \theta_{k m}$.

2. A high $X: R$ ratio is assumed ( $X$ : reactance, $R$ : resistance). Hence, $g_{k m}$ is very small compared to $b_{k m}$ and can be neglected.

3. An equal distribution of loads and power injections is assumed, so that $b_{k m}^{q}=0$.

4. All node voltages $U_{k}$ equal one in relation to the nominal node voltage (per unit system).

On the one hand, the simplifications lead to an easier solution process but, on the other hand, possibly also to results which are readily transferable to reality. The individual quality of approximation mainly depends on several network characteristics and is hard to be rated in general (cf. [12,26,27]).

Due to the assumptions, the reactive power flow $q_{\mathrm{km}}$ is set to zero and the active power flow $p_{k m}$ can be calculated in a linear expression as shown in Eq. (8). Additionally $p_{k m}=-p_{m k}$ can be assumed which means that all line losses are ignored.

$$
p_{k m}=b_{k m} \cdot \theta_{k m}
$$

The main advantage of DC models is that they are computationally inexpensive due to the linearity of the problem (cf. [24]). At present, they are primarily used for large energy system models which commonly are also modeled as linear programs. However, DC models only give approximations of the actual power flows in a network and completely neglect reactive power flows. Therefore, the validity of their application in energy system analysis has to be questioned.

For example, [27] analyzed the effect of the simplifications and found that the power flow calculated with a DC model is only a good approximation of the power flow calculated with an $\mathrm{AC}$ model, if there are no lines with a high reactive and a low active power flow. In [12] it is argued that, if certain network criteria are met, a DC power flow approximation is justified for techno-economic analysis. However, the problem is to ensure these network criteria.

In literature, several extension of DC models can be found which seek to overcome some major drawbacks of DC modeling. For example, additional restrictions ensure the consideration of power losses in Direct Current Models with Ohmic Losses (DCOL). A further approach is implemented in the so-called Relaxed DCOL (RDCOL) model which is proposed by [15]. It improves the solution process with an assumption based relaxation which leads to a mixed integer convex problem and hence the better applicability of the Branch and Bound algorithm [15]. However, each of these extensions leads to non-linear constraints, which is why a large part of the advantages in computing time of DC models gets lost. Hence, the approaches are mostly not directly suited for the application in large energy system models and necessitate new mathematical approaches to be gainfully integrated. 


\section{PERSEUS-NET: methodology and application}

\subsection{Model family PERSEUS}

The model family PERSEUS ('Program Package for Emission Reduction Strategies in Energy Use and Supply') comprises several optimizing bottom-up energy and material flow models, which were constantly developed in the recent decades [28-30]. The main focus of all PERSEUS models is to analyze the long-term development of supply systems within the considered system's boundaries (expansion planning) and to determine the realized unit commitment. Therefore, power supplying technologies are techno-economically modeled in detail while the power demand, which is the main model driver, is given exogenously. The target function is to minimize all system expenditures subject to the satisfaction of the given energy demand. The value of the objective function is the sum of all system relevant expenditures, discounted to the base year.

Like most bottom-up energy system models PERSEUS is also designed as an energy and material flow model, in which direct graphs are used to model sources and sinks as well as the energy and mass flows between them. This group of models is called transshipment models, being commonly used especially in energy system analysis. Regarding electric energy, networks are modeled in a very simplified way and physical constraints of power flows are neglected. Hence, power transfers from one region to another are modeled like the transport of any other commodity. Line losses are either neglected or approximated by fixed loss factors. Most of the models only incorporate a small number of regions. Within these regions power transfer is assumed to be perfect without losses or restrictions which led to the descriptive term of copperplate models.

Regarding the substantial structural changes of the energy system (cf. Sect. 1) which necessitate significant upgrades of energy models the first approach was to develop an energy system model which comprises a DC power flow model. The resulting model PERSEUS-NET $[7,8]$ and some illustrative results of its application are described in the following section.

\subsection{PERSEUS-NET}

The model PERSEUS-NET, as a further development of the models outlined in the previous section, is intended to find an optimal long-term expansion plan of power supply systems with respect to regional characteristics and power grid constraints. In addition to the comprehensive modeling of techno-economic characteristics of the power supply system, especially the location of existing infrastructure as well as regional characteristics in potential of renewable energy sources (RES) and power demand are implemented. To meet the challenges described, a hybrid modeling approach has been developed [7], consisting of

1. the bottom-up energy system model PERSEUS-NET, and

2. a geodatabase containing all relevant georeferenced input data.

PERSEUS-NET extends PERSEUS by a transmission network model and a DC optimal power flow model. As a result, besides the optimal future power plant mix, also 
the optimal topology of the power supply system can be determined. As decision criteria for a geographically optimal power plant expansion, the locational marginal costs of electricity supply are derived by using a locational marginal pricing approach based on [31]. The selection of a DC power flow model is due to its linearity. As PERSEUS is a linear optimization model, the integration of additional linear constraints is mathematically easy. However, the massively increased number of considered regions (due to the necessity of modeling the transmission grid), significantly increases the model size. Therefore, in the first step, the DC approach seems to be the most suitable method of considering network constraints in techno-economic energy system models. Nevertheless, further developments should comprise more precise load flow models in order to take the power grid into account more accurately.

\subsubsection{Locational marginal pricing in power markets}

To allow for a backcoupling of network characteristics to the process of investment decisions and unit committment, a locational marginal pricing (LMP) approach based on [31] is used. This approach takes account of regional characteristics by deriving individual prices at each considered node $k$ of the network. In the short-term LMP guarantees dispatching with respect to the physical laws of power flow and line flow constraints, which reduces costs of redispatch and voltage control. With a long-term time horizon, the LMP approach signalizes optimal time and especially optimal location of capacity investments. The time-dependent price $p_{k}(t)$ of supplying electricity at node $k$ with a time-dependent demand of $d_{k}(t)$ equals the marginal supply costs $C_{T o t}$ comprising operating as well as capital costs:

$$
p_{k}(t)=\frac{\partial C_{T o t}}{\partial d_{k}(t)}
$$

Due to the consideration of the whole energy system, the locational prices are calculated subject to generation and transmission constraints. According to economic theory, in a competitive market structure, marginal cost pricing is also optimal in a social welfare sense [32]. By minimizing the social costs of providing electricity to node $k$ in $t$ optimal locational prices can be obtained. These social costs $S C$ equal the sum of generator $G$ and network costs $N$ which themselves are functions of the generators' commitment $g(t)$ and the line flows $\mathbf{z}(t)$ minus customers' benefits $B$ depending on the demand $d(t)$ :

$$
S C=G[g(t)]+N[\mathbf{z}(t)]-B[d(t)]
$$

By minimizing (10) with respect to generation and network constraints and using the Lagrangian relaxation $[23,31]$, the optimal price $p_{k}(t)$ can be calculated as:

$$
p_{k}(t)=\mu_{e}(t)\left[1+\frac{\partial L}{\partial d_{k}(t)}\right]+\sum_{i} \mu_{i}^{Q S} \frac{\partial z_{i}(t)}{\partial d_{k}(t)}
$$


Hence, the optimal spot price $p_{k}(t)$ at node $k$ depends on the Lagrange multiplier of the energy balance constraint $\mu_{e}(t)$, the marginal costs of line losses, and on the Lagrange multiplier of line capacity constraints $\mu_{i}^{Q S}$.

Although the application of nodal or zonal pricing has been intensively investigated in research, and many theoretical contributions on potential design have been published, only few LMP concepts have been actually realized so far (e.g. in New Zealand or California).

\subsubsection{GIS-based representation of regional aspects}

According to the aim of respecting regional characteristics and network specifics in the techno-economic energy system model, a georeferenced data source is necessary. Using the software ArcGis (Esri) the transmission network data is prepared based on printed maps [33] and scans. As the data is stored within an Access database, ArcGis is directly compatible with the PERSEUS database. Moreover, the software allows for tailor-made visualization of the complex input and output data.

The most relevant geographical information in course of the development of PERSEUS-NET is the mapping of the transmission network and its grid nodes. Three different types of nodes are distinguished [7]:

- power plant nodes which represent grid nodes to which large power plants are connected,

- substation nodes at which substations of the transmission grid are installed, and

- county nodes representing administrative districts or counties.

The county nodes comprise also all georeferenced regional data such as the regional energy demands and RES potentials. The transmission grid itself is represented in the GIS (geographic information system) as the edges of a geographic network while all parallel lines are merged to one single line. It is to be noted, that only the transmission system is considered $(220 / 380 \mathrm{kV})$. In total, 441 grid nodes are implemented which are connected by 560 transmission lines. Thermal line limits and resistances are based on technical characteristics of typical power lines used in Germany [34].

\subsubsection{Further assumptions regarding the considered energy system}

The analysis is based on several further assumptions, which are crucial for the future development of Germany's energy system.

- Power demand: between 2010 and 2020 the power demand is assumed to be nearly constant in the range of 500-510 TWh/a. This is due to the combination of several effects working in opposite. On the one hand, the gross domestic product (GDP) increases, which leads to a rising demand which is, on the other hand, leveled out by a steadily decreasing population as well as increased energy efficiency. After the year 2020 the increase of GDP is even assumed to be overcompensated by the opposing effects. Hence, the assumed power demand in 2030 decreases to a level in the range of 490-500 TWh/a. The total demand is regionally distributed according to regional GDP and population forecasts (see Fig. 1a). 


\section{(A) Annual power demand [PJ]}

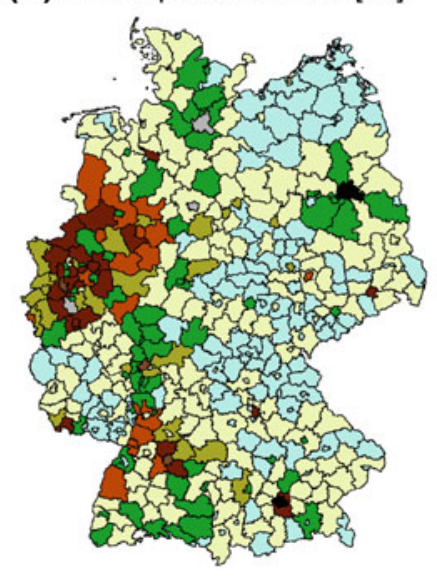

(B) Wind (onshore) - Installed Capacity [MW]
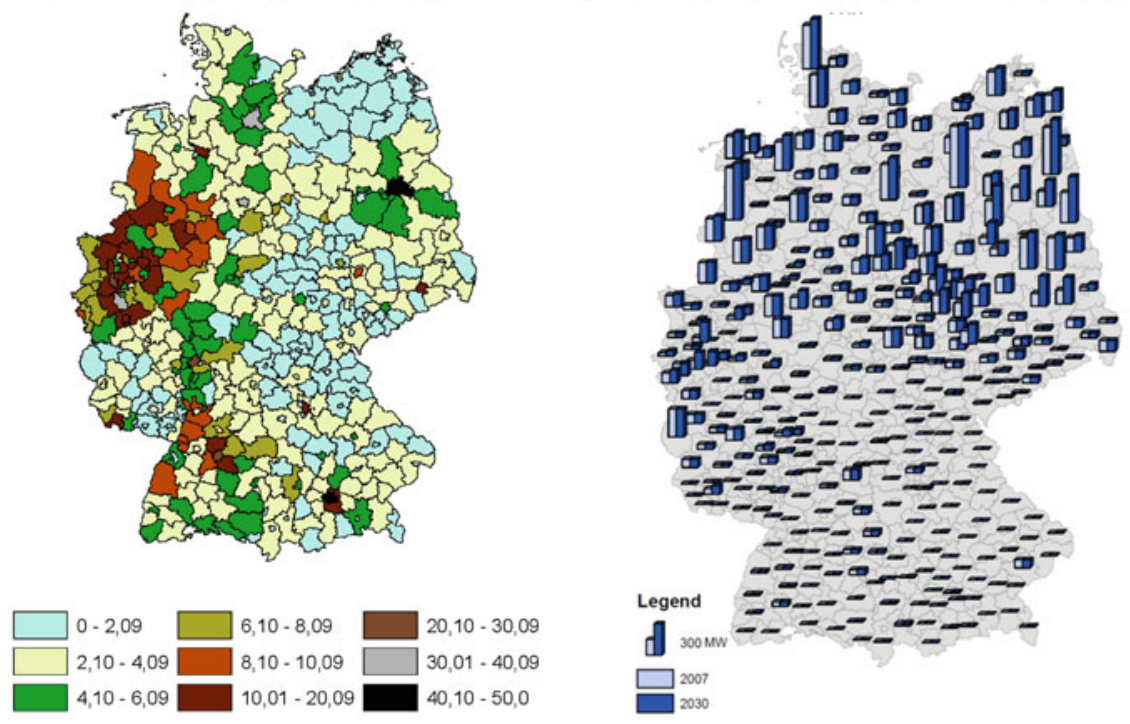

Fig. 1 Illustrative regional input assumptions: regional power demand (left) and regional wind onshore distribution (right)

- RES: electricity generation based on RES is intended to reach at least $30 \%$ by the year 2020 [35]. This goal is assumed to be reached and the regional distribution is based on calculations by [8] regarding the individual regional potentials (see Fig. 1b).

- Network expansion: the projects named in [36] are taken into account while further expansions are not assumed to be realized.

- Prices: the fuel price development is based on [37]. The carbon certificate prices are assumed to rise up to $45 € / t$ in year 2030 .

\subsection{Application and results}

Based on the assumptions described in the previous section, PERSEUS-NET is used to analyze the long-term development of Germany's power system. The development of power generation between 2007 and 2030 is strongly influenced by the predefined increase of the share of power generation from renewable energy sources, on the one hand, as well as by the policy induced phase-out of nuclear power stations. Due to its low generating costs, lignite power stations still have a dominant role in 2030. By contrast, the relevance of hard coal fired power stations decreases after 2020, while the share of gas fired power stations remains on an almost equal level between 2007 and 2030. Regarding the regional development of power generation, there is a significant shift of generation from the southern regions of Germany to the coasts (see Fig. 2). While in 2007 most power stations were situated close to the large demand centers or in the large lignite mining sides, power generation in 2030 is characterized by high 

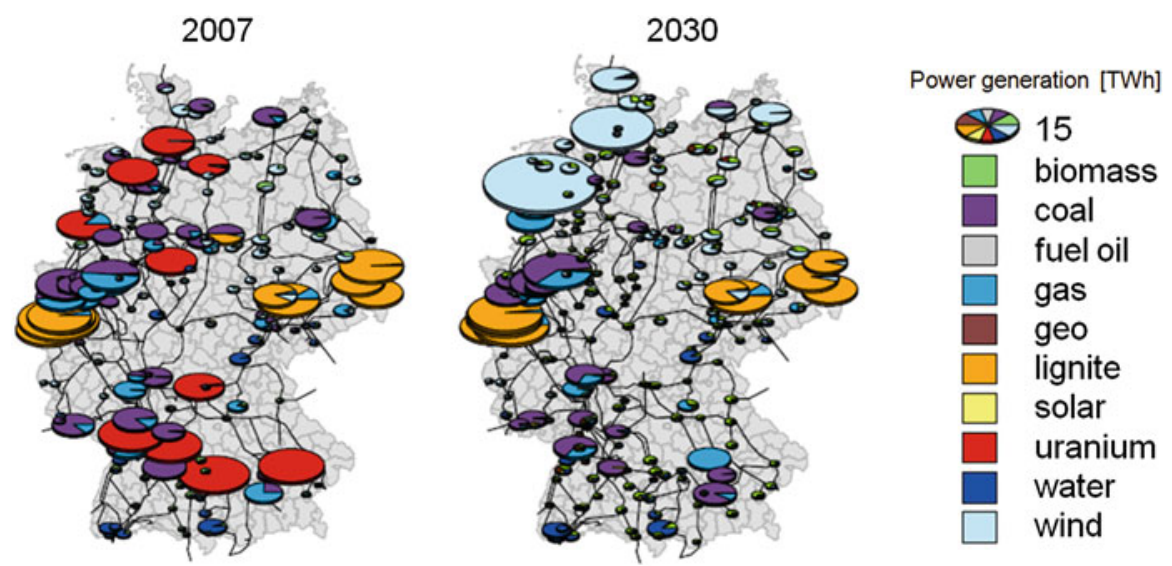

Fig. 2 Model results: shift of Germany's power generation from south to north [8]
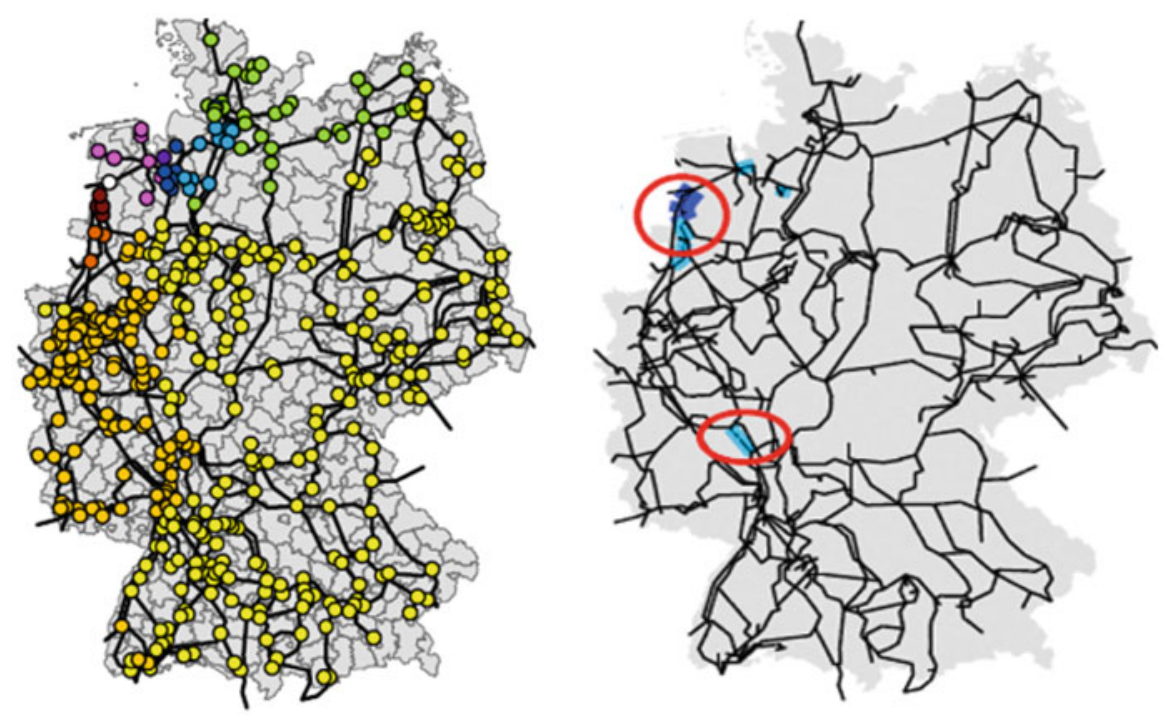

[EUR/MWh]

$\bigcirc(5,15] \bigcirc(55,65]$

$(15,25] \bigcirc(65,75]$

$(25,35] \bigcirc(75,85]$

- $(35,45] \bigcirc(85,95]$

$(45,55] \bigcirc(95,105]$

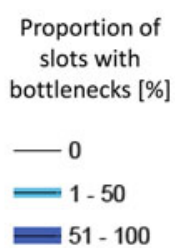

Fig. 3 Model results: nodal prices (left) and occurring bottlenecks (right) for the described scenario in 2030

offshore wind power feed-in. By 2030, power generation in Southern Germany is reduced by approximately 70 TWh compared to 2007 .

Regarding the situation in the German transmission grid, significant bottlenecks occur by 2030 (see Fig. 3 right). The most important bottleneck is situated in the 
northwest of Germany on power lines which are used to transmit offshore wind energy feed-in to the large load centers in the west and southwest of Germany.

As a result, significantly differing nodal prices occur in Germany by 2030 . Figure 3 (left) shows the regional distribution of average nodal prices in Germany in 2030. While the lowest nodal prices (around $5 € / \mathrm{MWh}$ ) can be found at the side of the bottleneck in the Northwest that has a generation surplus, the highest nodal prices (around $100 € / \mathrm{MWh}$ ) occur at the side of this bottleneck with a generation deficit. The average marginal cost of power supply increase over the covered time horizon from $38 € / \mathrm{MWh}$ in 2007 to $70 € / \mathrm{MWh}$ in 2030 . The main reasons for this increase are the scenario assumptions concerning $\mathrm{CO}_{2}$ certificate and fuel prices.

\subsection{Critical appraisal}

Compared with the still common use of pure transshipment models with few or even only one copperplate region in energy system modeling, the integration of a DC power flow model combined with a multi-regional structure is a major progress. PERSEUSNET takes the ongoing structural developments in the German energy system significantly better into account than former approaches. However, as previously mentioned, the DC power flow model applied in PERSEUS-NET still neglects line losses as well as reactive power. Therefore, the results are not directly transferable into reality. As described in [3], an AC approach should be used whenever network systemsespecially distribution grids - need to be analyzed in detail from a technical perspective. However, this still leads to problems in computing time and solvability due to the mathematically challenging structure, especially in techno-economic energy system models with a long-term time horizon. Hence, there is a need for new mathematical methods in energy system modeling under network constraints, which allow for an endogenous calculation of AC power flows (cf. Sect. 5).

\section{Modeling the distribution grid}

Aside the detailed modeling of the high voltage grid, the examination of the distribution grids plays a significant role. They serve to collect locally produced energy and transfer it to the high voltage transport grid, through which it gets delivered to the load centers. The most significant renewable energy source in the year 2011 was wind energy with an installed capacity of 29,100 MW and a produced electric energy of $48 \mathrm{TWh}$ [38].

Only about $10 \%$ of the approximately 22,300 installed wind power plants in Germany feed directly into the high voltage transportation grid $(220 / 380 \mathrm{kV})$. The majority, about $90 \%$, is connected to the superregional or regional distribution grids $(110 / 20 / 10$ $\mathrm{kV}$ ) [39]. In the same way, the bigger part of the other renewable energies is also integrated in the distribution grids, which emphasizes their importance and the need to examine them in energy system models in detail.

In the following, an analysis of the middle and low voltage grid of the municipality of Freiamt in Baden-Württemberg is presented. Freiamt is one of two model regions 
within the project MeRegio ${ }^{1}$ in whose framework extensive grid data was provided by the local grid administrator so that all grid equipment could accurately be remodeled with the commercial modeling software NEPLAN ${ }^{2}$.

The middle voltage grid $(20 \mathrm{kV}$ in this case) was completely modeled, with the exception of the feeding line from the distribution station Denzlingen $(110 / 20 \mathrm{kV})$ to the control unit Staudenhöfe. It comprehends 86 lines (overhead lines and buried cables) with a total length of $62.5 \mathrm{~km}$. Next to 73 local distribution stations there are 8 renewable energy feeders and one CHP plant mapped, feeding in to the middle voltage level.

At 68 local distribution stations, the underlying low voltage grid is mapped by summarizing loads and feedings. At the remaining 5 stations, the low voltage level was modeled in all detail down to the single household connections, which means 463 grid nodes, 396 lines with a total length of $23.5 \mathrm{~km}, 216$ loads and 71 feeders.

The aim of the analysis is to identify overloaded elements in the network and to demonstrate solutions for avoiding overloads. Furthermore a load flow analysis is done in order to identify the direction of the load flow. Two scenarios were calculated which are based on a static approach and which differ in their coincidence factors. In the first scenario (SCENARIO-HIGH) the coincidence factor is 0.6 and in the second (SCENARIO-LOW) it is 0.36. In both scenarios the injection of the decentralized capacities is between 70 and $100 \%$ of their installed capacity depending on the technology, e.g. $70 \%$ for wind power plants and $100 \%$ for small run-of-river power plants.

In both scenarios the direction of the load flow is not from the distribution station Denzlingen to the control unit Staudenhöfe but in the opposite direction. Even with a coincidence factor of 0.6 for the demand, the decentralized generation is higher than the demand, so electricity is re-injected to the distribution station Denzlingen. In both scenarios there are no overloaded assets in the network. The network is dimensioned in a way that all the decentralized generated electricity could be delivered to the distribution station. The maximum utilization of a power line in the low voltage grid is about $70 \%$, in the mid voltage grid $60 \%$ and in both voltage levels under $10 \%$ on average. These numbers show that the maximum of decentralized electricity generation is not reached and that more capacities could be integrated in the network without building new lines.

The next step should include a dynamic load flow analysis because this static approach is not sufficient enough to analyze dynamic effects in the grid. For this reason load and generation profiles have already been developed, which need to be integrated into the model. Subsequently, the calculations should be done with forecasted decentralized generation capacities, e.g. for the years 2020 and 2030 in order to analyze if the network is still able to handle the rising decentralized generation in the future. Especially the electricity generation from PV panels is rising very quickly and, consequently, the distribution grids could face problems. Thus, the importance of being able to evaluate different possibilities for removing potential grid congestions increases. However, NEPLAN does not allow for economic analyses or evaluations.

\footnotetext{
1 MeRegio: 'Aufbruch zu Minimum Emission Regions'. Project funded by the Federal Ministry of Economics and Technology (BMWi) as part of the 'E-Energy' project family - www.meregio.de.

2 NEPLAN Power System Analysis and Engineering - http://www.neplan.ch.
} 
Therefore, a new mathematical approach combining economic analysis and AC power flow modeling will be described in the following section.

\section{A new approach for AC-power flow modeling in energy system models}

Regarding the challenges identified in the previous sections, the long-term objective in research should be the development of a fully integrated and precise approach of power flow modeling in energy system models. Besides the importance of considering the high voltage grid, the increased relevance of decentralization also necessitates the integration of detailed information about middle and low voltage grids. Especially for voltage and power factor control, DC approaches are not sufficient due to their limitation to active power and the negligence of losses. However, pure AC modeling is not sufficient either since the economic aspects are missing as described in the previous section. Therefore, the aim is to develop an energy system model which integrates an AC power flow modeling approach.

\subsection{Modeling approach}

This section presents an outlook on a new mathematical method to meet the challenges described. Due to the high complexity as well as due to a lack of sophisticated data for the middle and low voltage grid, our first approach is to consider the highest voltage grid in a techno-economic energy system model. Hence, the model to be developed is intended to continue and improve the analysis described in Sect. 3 with the application of an AC power flow model.

The mathematical formulation and especially the numerical solution of an optimization problem with endogenous consideration of an $\mathrm{AC}$ power flow model is quite challenging. Due to the non-linear and non-convex structure of power flow constraints as described in Sect. 2.1, reliable estimates for global minima are hard to find. Moreover, the solution is numerically expensive due to the commonly high number of unknowns in large energy system models.

The non-linear and non-convex structure prevents the application of the methods used most commonly in energy economics, which are (mixed integer) linear programs. Hence, the first idea is to develop a soft coupling of two models:

1. An energy system model for the determination of an economically optimal solution for power plant dispatch and expansion planning, and

2. an electrotechnical power flow model for the calculation of the resulting power flows and the identification of network shortages due to the optimal power plant dispatch.

However, this approach has one major drawback: in case of the occurence of network congestions, the optimization has to be restricted accordingly. But this is hardly possible if no load flow restrictions are implemented, which themselves would require a model-endogenous load flow calculation. Therefore, in case of a network shortage and the lack of an integrated load flow calculation in the energy system model, besides penalty cost modeling only heuristic or knowledge-based methods remain as a 
possibility of adjusting the initially found optimal solution until the load flow is valid for the given network. Hence, two alternatives remain:

I. The complete integration of complex load flow restrictions in a techno-economic energy system model, which also requires the endogenous calculation of all occuring load flows, or

II. A partly decoupled iterative approach which enables a separate computation of power plant dispatch and load flows while still being mathematically coupled over specific coupling conditions.

The first alternative is suitable for very small problems, the second seems to be reasonable in case of real problems which are commonly very large and complex. In consequence, a new iterative algorithm was defined, meeting the challenges according to the second alternative. The complete mathematical definition can be found in our previous publication [3]. As a first step, both alternatives were implemented in a simplified version using $\mathrm{C}++$. Alternative II. gives rise to an iterative algorithm which enables the isolation of the non-linear and non-convex parts of the optimization in a separate step. For large-scale problems, the additional costs due to multiple operations are expected to be sufficiently smaller than solving the whole coupled optimization problem. First numerical experiments on a small test network have already shown promising results. However, proving the computational efficiency of large-scale problems compared to a coupled approach is subject to future research. It is to be noted, that the whole coupled optimization of large-scale problems might not be solvable at all due to its highly complex structure and memory restrictions which are expected to occur.

The following section describes some preliminary results we gained in an illustrative application of the new method. These results will be compared to a 'manual optimization' of the power plant dispatch combined with a power flow analysis in NEPLAN.

\subsection{Illustrative application}

For the validation of the modeling approach developed we implemented a small test energy system which represents the Upper Rhine Rift with five nodes. Some of the nodes are power producing nodes and hence representing the location of the power plants. Moreover, all nodes are modeled with an individual load which has to be covered by the power plants either directly or over the power grid. The grid is implemented according to the situation in reality. As the data situation for the highest voltage grid is quite good, all technical attributes, such as length and line impedances are available and can be integrated into the model. The test implementation seeks to prove the two following main aspects.

(i). The energy system model developed, which incorporates technical network constraints and hence the endogenous calculation of load flows for each considered line needs to prove its ability of the correct calculation of all complex load flows in the network. Therefore, all physical characteristics need to be represented correctly. 


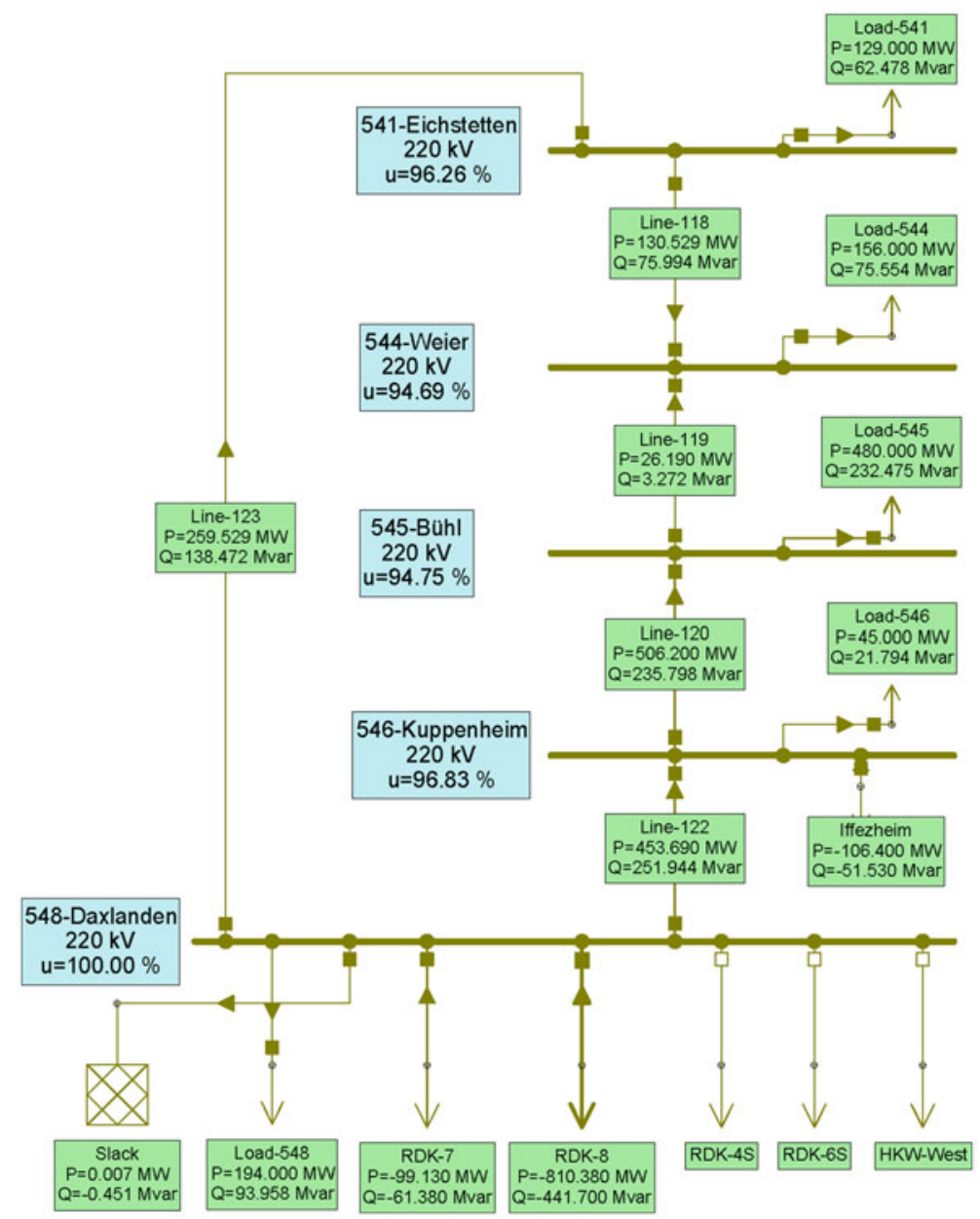

Fig. 4 Test model results in NEPLAN in situation (1)

(ii). The second aspect is to prove the ability of adapting the economically optimal solution of power plant dispatch in case of the occurrence of physical network constraints. This means, that if the virtually optimal solution causes a shortage in the power grid, the optimization model has to adjust its solution until the shortage is removed. Therefore, the model has to respect the complex interplay of power plant dispatch and the load flows in the power grid.

To prove (i) we implemented the test network both in our energy system model and in NEPLAN (cf. Fig. 4) with all technical characteristics. The electricity demand is exogenously given for each node of the network. All power producing processes are modeled according to their location and individual techno-economic characteristics. Hence, the optimization model with its target function of minimizing the total system expenses for covering the electricity demand, determines the optimal power plant dispatch for the given load situation. Furthermore, the resulting load flows are 
calculated endogenously. The optimal solution given by the optimization is now transferred into NEPLAN. Therefore, the determined power production of each individual power plant is modeled as negative load. In the next step, the calculation of the resulting load flows in NEPLAN shows, that the results of the optimization and the commercial electrotechnical software match perfectly. Also the calculated network losses (active and reactive) match, which proves the ability of our energy system model to correctly determine the load flows which result out of the economically optimal power plant dispatch.

The validation of aspect (ii) is more challenging. To determine the correct behavior of the model in case of the occurence of shortages in the power grid, three different network situations were simulated. The first one is congruent with the situation in (1) and represents the case of the feasibility of the optimal power plant dispatch. The second one incorporates several fictitious changes:

- Power plant Iffezheim is increased in its variable costs until the power plant dispatch optimization doesn't schedule it any more.

- As a consequence, line 118 has to carry more load than in the first situation: $s_{118}(1)=151.04 \mathrm{MW}, \quad s_{118}(2)=171.27 \mathrm{MW}$.

- Now, the capacity of line 118 cap $_{118}$ is restricted to a value between the two load situations:

$s_{118}(1)<\operatorname{cap}_{118}<s_{118}(2)$.

Each step was manually implemented in NEPLAN. As a result, an overload for line 118 is calculated as intended (cf. Fig. 5). However, the software is not able to give a cost optimal measure to remove this congestion.

In contrast to NEPLAN, the optimization model does not only detect the congestion in the power grid for the different situations but also determines the optimal solution for power plant dispatch with respect to the technical network constraints. In the artificially designed problem, the solution is to schedule the power plant Iffezheim again at its initial level. Even though the total costs for load coverage are higher due to the increase in variable cost, this is the optimal solution. It is correctly identified by the new approach. Hence, the model respects network constraints in an adequate way.

The third network situation is to restrict line 118 to a value $\operatorname{cap}_{118}<s_{118}(1)$ which means, that the initial optimal solution is not realizable. Due to the network design and the fact that only two of the five nodes are power plant locations even no valid solution exists. Also this case is computed correctly in our model because it states the problem as infeasible. With the given network characteristics and the exogenous load to be satisfied no valid solution exists.

\subsection{Critical appraisal}

Summarizing the illustrative application, the model developed proved its applicability of determining the economically optimal power plant dispatch with respect to technical network constraints for one exogenously given load situation. For the endogenous calculation of power flows a complete AC power flow model was integrated into the basic version of an energy system model seeking to cover the given electricity demand. Although the consideration of complex power flows is mathematically very 


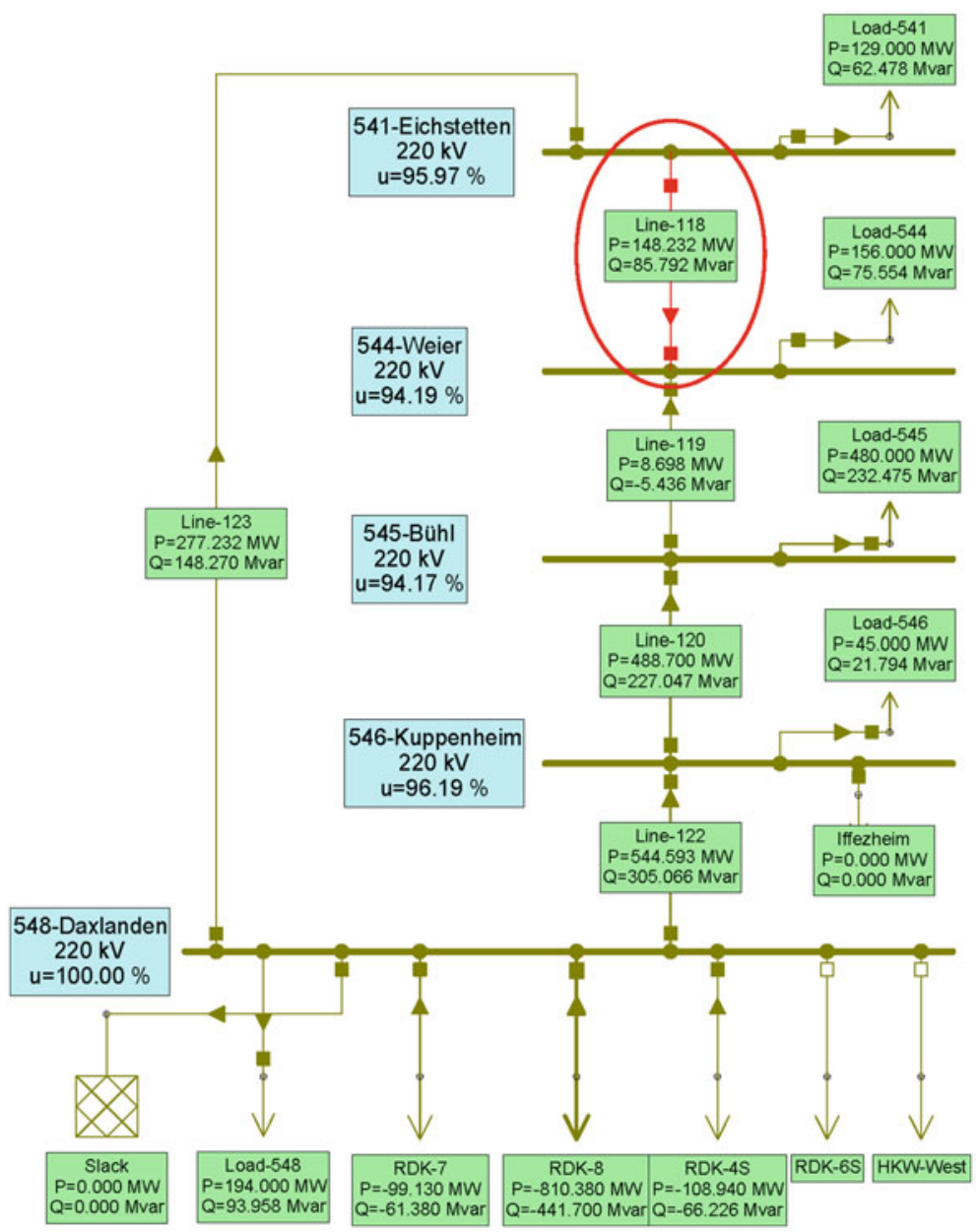

Fig. 5 Test model results in NEPLAN in situation (2)

challenging, the approach provided appropriate solutions and proved its correct reaction to network bottlenecks. However, the test network had only five nodes and not all techno-economic constraints were integrated in this first approach. Furthermore, only one discrete period was considered. Hence, the further development towards a comprehensive energy system model with a long-term time horizon is subject to further research.

\section{Conclusions}

The promotion of renewable energy sources (RES) and the increasing decentralization of energy systems brings about new challenges on all levels of the system. For instance, the grid load in the system is expected to rise to an extent that is hardly man- 
ageable with the existing grid capacities. Especially in Germany, the realization of the ambitious targets concerning the expansion of RES requires an extensive structural rearrangement of the system. Consequently, the importance of grid aspects for power system modeling will increase significantly. Therefore, different approaches for the consideration of grid constraints in energy system modeling were presented in this paper.

Firstly, for the analysis of the regional long-term development of the German power system as well as the occurrence of potential congestions in the transmission grid, a multi-period linear optimization model, comprising the system equations for power generation and transmission, was used. In comparison to the use of classical transshipment models with few or even only one copperplate region, the presented model constitutes a major progress. Secondly, since the increasing decentralization of electricity generation leads to an increasing occurence of backfeedings from lower to higher voltage levels, the usage of the AC power flow modeling tool NEPLAN for analyzing distribution grids was described. Thirdly, as NEPLAN does not allow for economic analyses, a new mathematical approach was presented, combining detailed AC power flow analysis with techno-economic energy system modeling. The application of the newly developed algorithm in a small test network showed the applicability of the concept. Since different power grids vary on a large scale in terms of structure, installed equipment, or number of installed renewable plants, it becomes apparent that each of the presented approaches is relevant to investigate specific questions considering particular restrictions.

Future research will be concentrated on the further development of the new technoeconomic energy system model with an integrated AC load flow model. The first aim is to expand the considered energy system to Germany and to integrate the highest voltage grid. Thus, the work will continue the analysis described in Sect. 3 on the basis of the novel approach described in Sect. 5. The latter proved to be applicable in a small test network for different network situations including the correct simulation of congestions as well as their economical optimal avoidance. One further step ahead, the results gained will be transferred to the middle and low voltage grid. Here, the first challenge will be to improve the deficient data situation and to develop methods to keep the data manageable, e.g. by considering various typical network sections as representatives for those in reality. Finally, it is doubtful whether it will be sufficient to analyze and evaluate possible strategies to avoid potential future grid congestions only with respect to economic aspects. Therefore, energy systems models will be combined with methods from the field of multi-criteria decision analysis (MCDA) in order to include technical, ecological but also socio-psychological (e.g. acceptance) factors in addition to economic criteria into the evaluation process of such strategies.

\section{References}

1. Haubrich, H.-J.: Technische Fragen beim Open Market Coupling (OMC). Study funded by the European Federation of Energy Traders and Verband der Elektrizitätswirtschaft e. V., Institut für Elektrische Anlagen und Energiewirtschaft; Forschungsgesellschaft Energie e. V., RWTH Aachen (2006)

2. Shustov, A.: Netzschutz für elektrische Energieversorgungssysteme mit hohem Anteil dezentraler Stromerzeugung. PhD thesis, Universität Kassel (2009) 
3. Schönfelder, M., Eßer-Frey, A., Schick, M., Fichtner, W., Heuveline, V., Leibfried, T.: New developments in modeling network constraints in techno-economic energy system expansion planning models. Zeitschrift für Energiewirtschaft 36(1), 27-35 (2012)

4. Frank, S., Steponavice, I., Rebennack, S.: Optimal power flow: a bibliographic survey I. Energy Systems, in press (2012)

5. Apfelbeck, J.: Simultane Optimierung des Kraftwerks- und Netzausbaus am Beispiel von Deutschland. VDI-Berichte 2080, 29-44 (2009)

6. Dietrich, K., Leuthold, F., Weigt, H.: Will the market get it right? The placing of new power plants in Germany. Zeitschrift für Energiewirtschaft 34, 255-265 (2010)

7. Eßer, A., Möst, D., Rentz, O.: Long-term power plant investment planning in Baden-Württemberg using a GIS-based nodal pricing approach. In: Proceedings of the 31st IAEE international conference "Bridging energy supply and demand: logistics, competition and environment" (2008)

8. Eßer-Frey, A., Fichtner, W.: Analyzing the regional development of the German power system using a nodal pricing approach. In: Proceedings of the 8th conference on the European electricity market (EEM) (2011)

9. Stamtsis, G., Christensen, J., Erlich, I.: Evaluation of power systems congestion using nodal price analysis. In: Proceedings of the international symposium MEPS, pp. 25-30 (2002)

10. Stigler, H., Todem, C.: Optimization of the Austrian electricity sector (control zone of VERBUND APG) under the constraint of network capacities by nodal pricing. Cent. Eur. J. Oper. Res. 13, 105-125 (2004)

11. Ding, F., Fuller, D.: Nodal, uniform, or zonal pricing: distribution of economic surplus. IEEE Trans. Power Syst. 20(2), 875-882 (2005)

12. Purchala, K., Meeus, L., Belmans, R.: Zonal network model of European interconnected electricity network (2005)

13. Weigt, H.: A time-variant welfare economic analysis of a nodal pricing mechanism in Germany. In: Proceeding of the 5th conference on applied infrastructure, research (2006)

14. Leuthold, F., Weigt, H., Hirschhausen, Chr v: Efficient pricing for European electricity networks-the theory of nodal pricing applied to feeding-in wind in Germany. Utilities Policy 16, 284-291 (2008)

15. Handschin, E., Kuhn, S., Rehtanz, C., Schultz, R., Waniek, D.: Optimaler Kraftwerkseinsatz in Netzengpasssituationen. In: Schultz, R., Wagner, H.-J., (eds) Innovative Modellierung und Optimierung von Energiesystemen, chapter 3, pp. 39-68. LIT Verlag Dr. W. Hopf (2009)

16. Duthaler, C.: Europe nodal: a simulation of the European electricity market based on the full network model. In: Second annual conference on competition and regulation in network industries, Center for European Studies (2009)

17. Barth, R., Apfelbeck, J., Vogel, P., Meiborn, P., Weber, C.: Load-flow based market coupling with large-scale wind power in Europe. In: 8th workshop on large-scale integration of wind power into power systems as well as on transmission networks for offshore wind farms (2009)

18. Waniek, D., Rehtanz, C., Handschin, E.: Flow-based evaluation of congestions in the electric power transmission system. In: EEM 2010-7th conference on the European, energy market (2010)

19. Leuthold, F., Weigt, H., Chr. v. Hirschhausen.: A large-scale spatial optimization model of the European electricity market. Networks and spatial economics, pp. 1-31 (2010)

20. Leuthold, F.: Economic engineering modeling of liberalized electricity markets: approaches, algorithms, and applications in the European context. Technische Universität Dresden (Dissertation), Dresden (2010)

21. Weigt, H., Jeske, T., Leuthold, F., Hirschhausen, Chr v: Take the long way down: integration of largescale North Sea wind using HVDC transmission. Energy Policy 38, 3164-3173 (2010)

22. Stamtsis, G.: Power transmission cost calculation in deregulated electricity market. Logos, Berlin (2004)

23. Green, R.: Electricity transmission pricing: how much does it cost to get it wrong? CMI Working Paper (2004)

24. Powell, L.: Power system load flow analysis. McGra-Hill, New York (2004)

25. Sun, J., Tesfatsion, L.: DC optimal power flow formulation and solution using QuadProgJ_Department of Economics Working Paper Series, vol. 06014. Iowa State University, Iowa (2006)

26. Murillo-Sánchez, C., Thomas, R.: Thermal unit commitment with a nonlinear AC power flow network model. In Hobbs, et al., (eds) The next generation of electrical power unit commitment models, chapter 5, pp. 75-92. Kluwer Academic Publishers, Dordrecht (2001) 
27. Overbye, T., Cheng, X., Sun, Y.: A comparison of the AC and DC power flow models for LMP calculations. In: Proceedings of the 37th Hawai international conference on system science (2004)

28. Enzensberger, N.: Entwicklung und Anwendung eines Strom- und Zertifikatmarktmodells für den europäischen Energiesektor. VDI Verlag, Düsseldorf (2003)

29. Möst, D.: Zur Wettbewerbsfähigkeit der Wasserkraft in liberalisierten Elektrizitätsmärkten—Eine modellgestützte Analyse dargestellt am Beispiel des schweizerischen Energieversorgungssystems. Peter Lang, Frankfurt a.M. (2006)

30. Rosen, J.: The future role of renewable energy sources in European electricity supply: a model-based analysis for the EU-15. Universitätverlag Karlsruhe, Karlsruhe (2007)

31. Schweppe, F., Caraminis, M., Tabor, R., Bohn, R.: Spot pricing of Electricity. Kluver Academic Publishers, New York (1987)

32. Stoft, S.: Power system economics-designing markets for electricity. IEEE-Press, WileyInderscience, New York (2005)

33. UCTE.: Map of the interconnected network of the UCTE. UCTE - printed publications (2006)

34. Kiessling, F., Nefzger, P., Nolasco, J., Kaintzyk, U.: Overhead power lines: planning, design, construction. Springer, Berlin (2003)

35. BMU.: Weiterentwicklung der Ausbaustrategie Erneuerbare Energien-Leitstudie 2008, Bundesministerium für Umwelt. Naturschutz und Reaktorsicherheit, Berlin (2008)

36. EnLAG.: Gesetz zur Beschleunigung des Ausbaus der Höchstspannungsnetze vom 21. August 2009. BGB1, I(55):2870-2876 (2009)

37. IEA.: World energy outlook. International energy agency (2008)

38. Bundesverband WindEnergie e.V. online published data on wind power production (2012) http://www. wind-energie.de/ (access: 20.04.2012)

39. Pfeiffer, K., Schwarz, H.: Netzengpässe in Verteilnetzen und technische Lösungsmöglichkeiten. uwf - UmweltWirtschaftsForum 17(4):345-349 (2009) 


\section{Repository KITopen}

Dies ist ein Postprint/begutachtetes Manuskript.

Empfohlene Zitierung:

Nolden, C.; Schönfelder, M.; Eßer-Frey, A.; Bertsch, V.; Fichtner, W.

Network constraints in techno-economic energy system models: towards more accurate modeling of power flows in long-term energy system models.

2013. Energy systems, 4.

doi:10.554/IR/1000045256

Zitierung der Originalveröffentlichung:

Nolden, C.; Schönfelder, M.; Eßer-Frey, A.; Bertsch, V.; Fichtner, W.

Network constraints in techno-economic energy system models: towards more accurate modeling of power flows in long-term energy system models.

2013. Energy systems, 4 (3), 267-287.

doi:10.1007/s12667-013-0078-0 